\title{
FITKIT ANDROID APPLICATION
}

\author{
Aashita Chhabra, Chitrank Tyagi \\ Department of Information Technology, \\ Guru Gobind Singh Indraprastha University, \\ Sector 16C, Dwarka, Delhi, 110078
}

\begin{abstract}
Age is just a number" a quote that explains one can never get old if one follows proper healthy routine. With the initiatives taken by the government and various industries (Bollywood, Cricket etc) fitness is gaining popularity among the individuals. Smartphones and tablets are slowly but steadily changing the way we look after our health and fitness. Today, many high quality mobile apps are available for users and health professionals and cover the whole health care chain, i.e. information collection, prevention, diagnosis, treatment and monitoring. Our team has developed a mobile application called FitKit which is implemented using Android framework and is available for android users. Our app mainly focuses on fitness regimes inclusive of meals allotment, workout routine, chat support with experts from the fitness enthusiasts and tracking fitness activities like steps counter. Nowadays people ranging from teenage to adults i.e., from college students to even people in their old ages are becoming fitness freaks and are more concerned regarding their diets and fitness.
\end{abstract}

Keywords - Fitness, Android Application, Firebase, Steps Counter, Diet Chart, Workout Routine

\section{INTRODUCTION}

"All progress takes place outside the comfort zone." For a healthy life one has to plan everything in an organized manner i.e., placing meals at regular intervals, doing regular workout and exercises.

Fitness enthusiasts who follow fitness regimes uses various apps for tracking fitness activities like using workout routine app for designing their workout, using apps for designing their meals , using apps for tracking fitness like steps count and many people listen to music while doing workout hence using a separate app. FitKit provides all these features under one application only. Further our app also provides a chat with expert feature where one can ask for help regarding meals, designing custom workout routine and workout supplements. Our app uses the concept of BMI(Body Mass Index) for determining meals and workout. BMI is a person's weight in kilograms $(\mathrm{kg})$ divided by his or her height in meters squared. The National Institutes of Health (NIH) now defines normal weight, overweight, and obesity according to BMI rather than the traditional height/weight charts.

\section{A. Application Fundamentals:}

Android applications are written in Java programming language. However, it is important to remember that they are not executed using the standard Java Virtual Machine (JVM). Instead, Google has created a custom VM called Dalvik which is responsible for converting and executing Java byte code. All custom Java classes must be converted (this is done automatically but can also be done manually) into a Dalvik compatible instruction set before being executed into an Android operating system. Dalvik VM takes the generated Java class files and combines them into one or more Dalvik Executable (.dex) files. It reuses duplicate information from multiple class files, effectively reducing the space requirement (uncompressed) by half from a traditional .jar file. Dalvik was created to support the nature of lightweight mobile operating systems require because of the limited hardware capabilities compared to conventional desktops or laptops.

\section{LITERATURE SURVEY}

Several applications have been developed regarding fitness. Applications like HealthyfyMe, GoogleFit are some of the popular apps related to fitness.

HealthyfyMe uses calorie counter, allocates diet plan, food tracker \& calculator, eat healthy, lose weight. It aims at solving problems related to weight loss. Developed for both Android and iOS platforms, the application provides calorie tracking, water tracking and on-the-cloud fitness coaching. In addition to these, the app takes the gamified approach to keep users motivated.

HealthifyMe works on a freemium model.

Google Fit is a health-tracking platform developed by Google for the Android operating system, Wear OS and Apple Inc.'s IOS. It is a single set of APIs that blends data from multiple apps and devices. It uses the sensors built into your device to automatically track activities like walking, biking and running. You can also use it to keep track of your fitness goals and weight-loss progress over the past day, week and month. The Google Fit app is available as a free download in the Play store.

Live a healthier, more active life with Fitbit, the world's leading app for tracking all-day activities, workouts, sleep and more. ... RECORD WORKOUTS: Use your Fitbit tracker to track your exercise, then check the app to see your stats, their impact on your day, and how your performance is improving. 


\section{International Journal of Engineering Applied Sciences and Technology, 2019 \\ Vol. 4, Issue 4, ISSN No. 2455-2143, Pages 203-205 \\ Published Online August 2019 in IJEAST (http://www.ijeast.com)}

Nike Training Club helps you reach your fitness goals. Get fit anytime, anywhere with free workouts across strength, endurance, yoga \& mobility. ... Nike Training Club members love our short, bodyweight only workouts to get the results they want even when life gets in the way.

\section{METHODOLOGY}

FitKit uses the concept of BMI for allocating workout and designing meals routine. BMI is calculated using the formula shown in fig. 1.

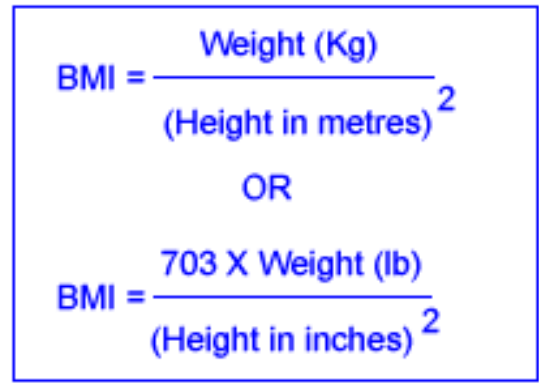

Fig. 1 BMI Formula

Further BMI is studied on the basis of four categories underweight, normal, overweight, obese.

TABLE I

BMI CATEGORIES

\begin{tabular}{|l|l|}
\hline Weight Status Category & BMI Range \\
\hline Heavyweight & Below 18.5 \\
\hline Obese & 18.5 to 24.9 \\
\hline Normal & 25 to 29.9 \\
\hline Underweight & Above 30 \\
\hline
\end{tabular}

Based on the categories mentioned in Table 1 our app shows to which category our user belongs to and then responds accordingly. However Steps Counter uses the concepts of sensors which can work if we place phone in our pockets or at any wrist bands so that it can sense our motion while we are moving because if we are going to place the phone in our bag or at any still position then our phone sensors won't work effectively. Meals will be allotted to users using the net calorific values of each item which will be then compared with the required calorific value content value which one must follow for being healthy and fit.

\section{TECHNOLOGY}

FitKit app is designed AndroidOS platforms and is designed using Android framework. We have implemented application using Android Studio which serves as IDE for android. As we know, Android is java based framework and uses XML for frontend development. We have used the concepts like fragments, view pagers, floating action buttons, menu, splash screen etc. We have used Firebase Server for authentication and chat support. Firebase is a mobile and web application development platform developed by Firebase, Inc. in 2011, then acquired by Google in 2014. As of October 2018, the Firebase platform has 18 products, which are used by 1.5 million apps.

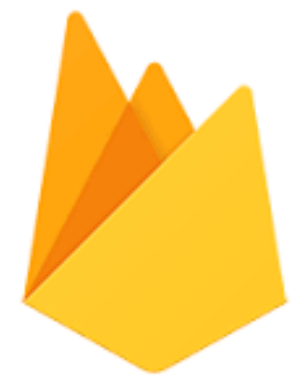

Fig. 2 Firebase

Firebase provides us a powerful tool, Firebase Authentication is most secured authentication among all servers like Php, Django. It provides us a concept of using Google Authentication, Email Verification, OTP based Mobile authentication and many more. Chat support is carried out using Real time Database and using database storage for sending pictures. For sending pictures over chat application we have used Glide library of github. For implementing the concept of Steps Counter we have used sensors and sensor manager. Steps Counter can work even when the app is in the background state.

\section{FUTURE SCOPE}

There is always a scope of improvement in every application or industry practice that is carried out in the world however the need is to keep the practice as simple as possible so that even a non-technical user who doesn't have the technical knowledge regarding the field can adopt our practice.

In the future we can implement the concept of workout playlists in FitKit so that the retention of our app can improve. As we know about $90 \%$ of the people listen to music while doing workout so as of now user will use our app only when he/she is doing workout or when they want to check their meals but if we want to improve our app retention we can implement workout playlists so that whenever the person is doing workout they can use our app for listening music also thereby increasing the overall retention percentage of our app. We can involve various fitness enthusiasts to collaborate with us and work as an expert, so that they can guide our users more efficiently.

Also we can implement the concept of tracking user's record over the app so that he/she can compare their progress over 


\section{International Journal of Engineering Applied Sciences and Technology, 2019 \\ Vol. 4, Issue 4, ISSN No. 2455-2143, Pages 203-205 \\ Published Online August 2019 in IJEAST (http://www.ijeast.com)}

time. After implementing this feature one can decide their goals accordingly.

\section{CONCLUSION}

Few numbers of motivations has driven this research. FitKit application is designed to cater all the demands related to fitness like workout routines, diet allotment and tracking fitness activities like steps counter. Our app focuses more on developing core strengths by giving every individual the required maintenance range for Calories one the basis of their BMI. It is designed in such a way that even those having not sufficient knowledge of the domain can easily use it as its UI is very simple. Overall FitKit can guarantee of taking responsibility of each individual's fitness and healthy lifestyle.

\section{ACKNOWLEDGEMENT}

I would like to take the opportunity to acknowledge the support and help of all who have assisted me in the research. Without their contribution and advice, I would have never been able to progress with the work in the research. Firstly, I would like to sincerely acknowledge my research mentor Ms. Aashita Chhabra, for her guidance, support, technical knowledge and encouragement in the whole research process and work. The information and feedback provided were extremely helpful and useful for designing of mobile application.

\section{REFERENCES}

[1] Dawidowicz, Paula 2010 Literature Reviews Made Easy: A Quick Guide to Success. IAP

[2] Gargenta, Marko 2011 Learning Android. O’Reilly Media, Inc.

[3] ACSM Journal for Fitness and Workout Routines accessed on 19.6.2019.

[4] Diet ,Nutrition and Workout related information from www.bodybuilding.com, www.flex.com accessed on 19.6.2019

[5] Android Related Information from http://developer.android.com/guide accessed on 15.6.2019.

[6] Hello Android, Introducing Google Mobile Application Development Platform, ,Ed Burnette, Fourth Edition.

[7] Developing Web Applications Using Firebase by https://firebase.google.com/docs accessed on 15.6.2019.

[8] Goldberg, Kevin 2009 Visual QuikStart Guide. Berkeley, CA 94710 How to Display List of Images in ListView in Android? http://stackoverflow.com/questions/459729/how-to-displaylist-of-images-in-listviewin-android, accessed November 4, 2013.

[9] Jordan, Lucas, and Pieter Greyling 2011 Practical Android Projects. Apress.
[10] Laycock, G. T. (1993). The Theory and Practice of Specification Based Software Testing. Dept of Computer Science, Sheffield University, UK.

[11] Lee, B. W.-M. (2012). Android Application Development Cookbook. In Android Application Development Cookbook: 93 Recipes for Building Winning Apps. Indiana: John Wiley \& Sons, Inc.

[12] Parse. (n.d.). Parse documentation. Retrieved 2013, from Parse: https://parse.com/docs/android_guide

[13] St. Laurent, Andrew M. (2008). Understanding Open Source and Free Software Licensing. O'Reilly Media.

[14] CnetJRE http://download.cnet.com/Java-RuntimeEnvironment-JRE/3000-2378_4-

10009607.html\#ixzz2mLmJ28Kg accessed on 12.2.2019.

[15] Android Architecture 2019[R/OL]. http://www.cnmsdn.com/html/201003/1268713218ID2058_2 .html 